\title{
A SUMMARY OF RADIO PULSAR POLARIZATION
}

\author{
C. $\mathrm{ZHU}$ \\ Xinjiang Astronomical Observatory, Chinese Academy of Sciences \\ 150 Science-1 Street, Urumqi, Xinjiang 830011, China \\ Graduate University of Chinese Academy of Sciences, 19A Yuquan Road \\ Beijing 100049, China \\ zhucui@xao.ac.cn \\ W. M. YAN* and N. WANG ${ }^{\dagger}$ \\ Xinjiang Astronomical Observatory, Chinese Academy of Sciences \\ 150 Science-1 Street, Urumqi, Xinjiang 830011, China \\ *yanwm@xao.ac.cn \\ ${ }^{\dagger}$ na.wang@xao.ac.cn
}

\begin{abstract}
Pulsars are one of the most polarized radio sources in the universe. Polarization measurements of pulsars provide us a lot of information, not only about the emission process itself, but also about the interstellar medium. This article describes the polarization properties of radio pulsars in general and discusses the observed rotation measure variations with time in pulsars.
\end{abstract}

Keywords: Pulsars; polarization; interstellar medium.

PACS numbers: 97.60.Gb, 95.30.Gv, 98.90.+s

\section{Introduction}

Pulsars are rapidly rotating highly magnetized neutron stars, which were discovered in $1967 .{ }^{1}$ It has been observed that radio emission from pulsars is highly polarized. The polarization properties of a pulsar are very important for understanding the pulse emission mechanism and the geometry of the system.

The observed pulsar radiation is affected by various propagation effects. Faraday rotation is relatively easy to observe in pulsars, which is described by the rotation measure (RM), defined by

$$
\psi=\mathrm{RM} \lambda^{2}
$$

where $\psi$ is the linear polarization position angle (PA) and $\lambda$ is the radio wavelength. The rotation measure is given by

$$
\mathrm{RM}=0.810 \int_{0}^{D} n_{e} \boldsymbol{B} \cdot d \boldsymbol{l}
$$




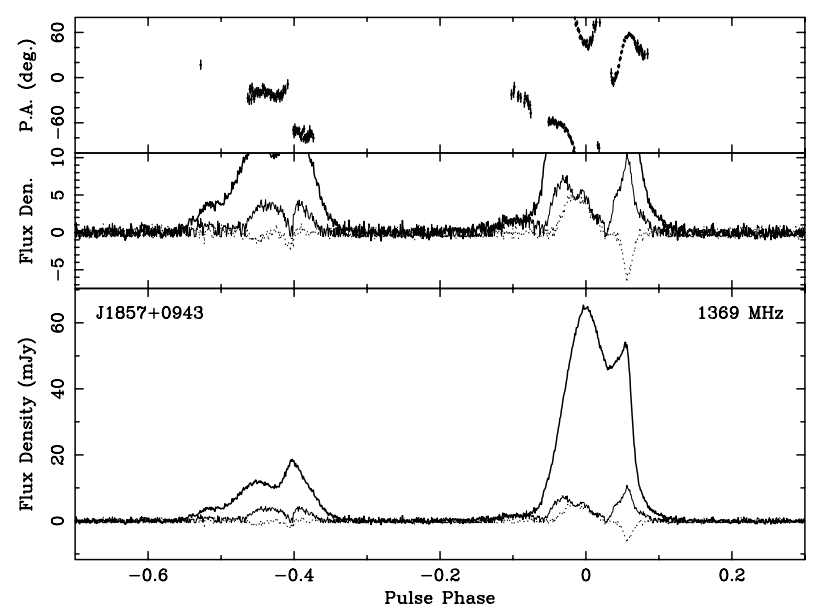

Fig. 1. Polarization profiles for PSR J1857+0943 at $1369 \mathrm{MHz}$ observed at Parkes (data from Ref. 4). The lower part gives the pulse profile for total intensity $I$ (thick solid line), linearly polarized intensity $L$ (thin solid line), and circularly polarized intensity $V$ (dotted line). The middle part is an expanded plot showing low-level details of the polarization profiles and the upper part gives the position angle of the linearly polarized emission.

where $n_{e}$ is the interstellar electron density in units of $\mathrm{cm}^{-3}, \boldsymbol{B}$ is the vector magnetic field in microgauss, $d \boldsymbol{l}$ is an elemental vector along the line-of-sight toward us (positive RMs correspond to fields directed towards us) in parsecs and RM is in units of $\mathrm{rad} \mathrm{m}^{-2}$. Dispersion occurs when the pulsar signal propagates through the interstellar medium (ISM), which is described by the dispersion measure (DM)

$$
\mathrm{DM}=\int_{0}^{D} n_{e} d l,
$$

where DM is expressed in units of $\mathrm{cm}^{-3}$ pc. Combining Eq. (2) and (3), we obtain an estimation for the mean line-of-sight component of the interstellar magnetic field:

$$
\left\langle B_{\|}\right\rangle=1.232 \frac{\mathrm{RM}}{\mathrm{DM}} \mu \mathrm{G} .
$$

Thus, pulsar polarization contributes to measure the structure of our Galactic magnetic field. ${ }^{2}$

Temporal variations in RM were observed in some pulsars. RM changes occur as the path to the pulsar traverses different regions of ISM or in the Earth's ionosphere due to the diurnal and other changes in the ionospheric total electron content (see Eq. (2)).

\section{Polarization Profile Properties}

In pulsar astronomy, millisecond pulsars (MSPs) are thought to be a different population from normal (non-millisecond) pulsars. Because in many respects, MSPs 


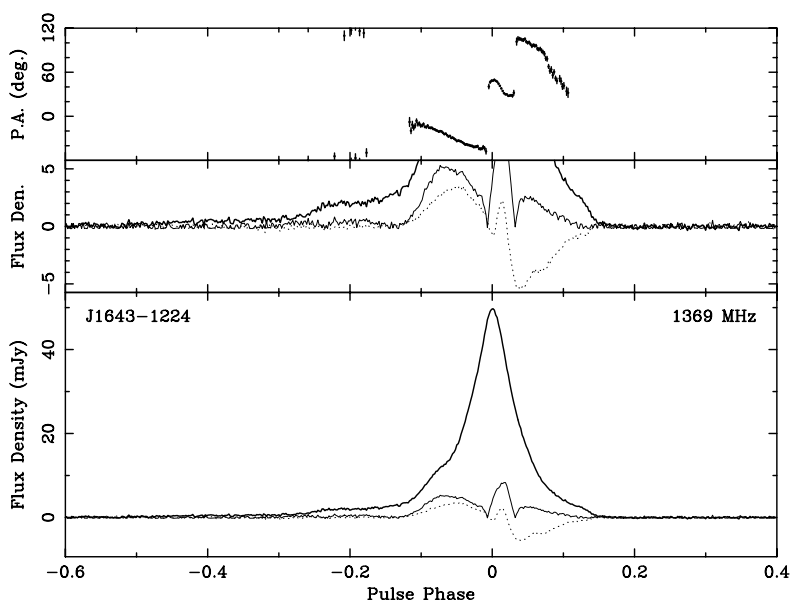

Fig. 2. Polarization profiles for PSR J1857+0943 at $1369 \mathrm{MHz}$ observed at Parkes (data from Ref. 4). See Fig. 1 for further details.

have very different properties to those of normal pulsars. Compared with normal pulsars, MSPs have shorter periods, smaller period derivatives, larger characteristic ages and weaker implied dipole magnetic fields. Despite these differences, the polarization characteristics of MSPs are similar to those of normal pulsars - they have similar high degrees of linear polarization, orthogonal mode PA jumps are observed and circular polarization with sense changes near the profile center is seen in both classes.

In many normal (non-millisecond) pulsars, the form of the observed linear polarization position angle (PA) variations with pulse phase is the characteristic S-shape, which can be approximately described by the 'rotating vector model' (RVM). ${ }^{3}$ However, usually in MSPs, the observed PA variations across their wide profiles are incredibly more complex, and are not even approximately described by the RVM. ${ }^{4}$ Figure 1 is an example to show the complexity of PA variations for millisecond pulsars. In PSR J1857+0943, the pulse profile shows an interpulse separated by about $180^{\circ}$ from the main pulse. Only less than 2 per cent of normal pulsars show interpulse emission $^{5}$ and most of this 2 per cent have short periods and high spin-down luminosities. However, interpulses are much more common in MSPs, with more than 60 per cent of the sample in Ref. 4 showing this features.

Not all pulsars show smooth and continuous PA swings. Discontinuities of approximately $90^{\circ}$ can be observed in some pulsars, and these are interpreted as resulting from overlapping emission from orthogonally polarized emission modes. This feature can be observed in both normal pulsars and MSPs, but MSPs more often show orthogonal mode PA jumps. Figure 2 is an example to show orthogonal mode PA jumps observed in a MSP. 


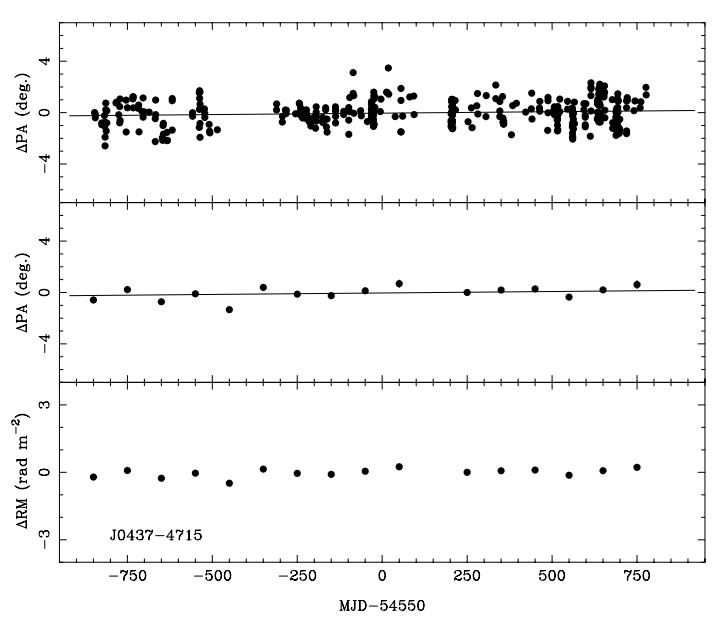

Fig. 3. PA variations for PSR J0437-4715 observed at Parkes (data from Ref. 11). The upper panel has the IRI-corrected PA variations showing the fitted linear trend. The middle panel shows the same data averaged over 100-day blocks with the fitted line as in the upper panel. The lower panel shows the smoothed variations converted to RM changes using Eq. (1).

Reference 4 detected previously unknown profile features in many pulsars because of the relatively high $\mathrm{S} / \mathrm{N}$ of their polarization profiles. The newly detected profile features greatly extend the range of pulse phase over which emission is detected.

For MSPs, the observed complex PA variations generally make single-axis RVM fitting impossible and caustic deformations and multiple emission regions dominate the observed pulse profile. ${ }^{4}$ None of the "standard" methods determining the heights of emission-region are applicable to many (probably most) MSPs.

\section{Rotation Measure Variations}

RM and DM variations with time were observed for some pulsars. Ref. 6-7 analyze the variations of RM and DM for the Vela pulsar. They interpret the temporal RM and DM variations for the Vela pulsar as the result of the relative motion of a magnetized filament of the Vela supernova remnant out of the line-of-sight. Following this interpretation, they estimated the electron density and the effective mean line-of-sight component of magnetic field in the filament (see Eq. (1)). Ref. 8 made a detailed interval to interval analysis of the RM and DM variations for the Crab pulsar. Several other authors ${ }^{9-10}$ also present some pulsars showing variations in their RMs.

Reference 11 studied RM variations with time for 20 millisecond pulsars observed at Parkes with correction for Faraday rotation in the Earths ionosphere. They found that the observed variations are dominated by changes in the Faraday rotation occurring in the Earths ionosphere. Fig. 3 is an example to show the RM variations in PSR J0437-4715. 


\section{Conclusions}

In general, normal pulsars and MSPs have very similar observed polarization properties, although usually MSPs have more complex pulse profile shapes and PA swings. However, wide pulse profiles, interpulses and orthogonal mode PA jumps are more common in MSPs. These imply that the basic radio emission mechanism is the same in both classes of pulsar, but the emission region for MSPs is more complex, probably consisting of several quasi-independent emission zones.

$\mathrm{RM}$ variations with time is common in pulsars. This may result from changes of ISM on the path to the pulsar. No significant long term RM temporal variations were observed in millisecond pulsars. The changes in the Faraday rotation occurring in the Earths ionosphere may dominate the observed RM variations.

\section{Acknowledgments}

This work was supported by NSFC projects 10903019, 11003034 and 11173041, National Basic Research Programme of China (973 Programme 2009CB824800), the West Light Foundation of Chinese Academy of Sciences under grant No. XBBS200920 and XBBS201021, and the Key Laboratory of Radio Astronomy, Chinese Academy of Sciences.

\section{References}

1. Hewish, A., Bell, S. J., Pilkington, J. D. H., Scott, P. F. and Collins, R. A. Nature, 217 (1968) 709.

2. Han, J. L., Manchester, R. N., Lyne, A. G., Qiao, G. J. and van Straten, W. ApJ, 642 (2006) 868.

3. Radhakrishnan, V. and Cooke, D. J. Astrophys. Lett., 3 (1969) 225.

4. Yan, W. M., Manchester, R. N., van Straten, W., Reynolds, J. E., Hobbs, G., Wang, N., Bailes, M., Bhat, N. D. R., Burke-Spolaor, S., Champion, D. J., Coles, W. A., Hotan, A. W., Khoo, J., Oslowski, S., Sarkissian, J. M., Verbiest, J. P. W. and Yardley, D. R. B. MNRAS, 414 (2011) 2087.

5. Weltevrede P., Johnston S. MNRAS, 387 (2008) 1755.

6. Hamilton, P. A., McCulloch, P. M., Manchester, R. N., Ables, J. G. and Komesaroff, M. M. Nature, 265 (1977) 224.

7. Hamilton, P. A., Hall, P. J. and Costa, M. E. MNRAS, 214 (1985) 5.

8. Rankin, J. M., Campbell, D. B., Isaacman, R. B., and Payne, R. R. Astron. Astrophys., 202 (1988) 166.

9. Han, J. L., Manchester, R. N., and Qiao, G. J. MNRAS, 306 (1999) 371.

10. Weisberg, J. M., Cordes, J. M., Kuan, B., Devine, K. E., Green, J. T., and Backer, D. C. ApJS, 150 (2004) 317.

11. Yan, W. M., Manchester, R. N., Hobbs, G., van Straten, W., Reynolds, J. E., Wang, N., Bailes, M., Bhat, N. D. R., Burke-Spolaor, S., Champion, D. J., Chaudhary, A., Coles, W. A., Hotan, A. W., Khoo, J., Oslowski, S., Sarkissian, J. M., Yardley, D. R. B. Ap\&SSS, 335 (2011) 485. 